\title{
BOOLEAN POWERS OF GROUPS
}

\author{
JOHN LAWRENCE ${ }^{1}$
}

\begin{abstract}
A group is $B$-separating if a Boolean power of the group has a unique Boolean algebra. It is proved that a finite subdirectly irreducible group is $B$-separating if and only if it is non-Abelian.
\end{abstract}

Suppose $B$ is a Boolean ring and $G$ is a group. Let $B[G]$ denote the group ring of $G$ with coefficient ring $B$. The Boolean power $G[B]$ is defined to be the set of those elements

such that

$$
\sum e_{i} g_{i} \in B[G]
$$

(1) $\sum e_{i}=1$,

(2) $e_{i} e_{j}=0$ if $g_{i} \neq g_{j}$.

The support of the element is the set $\left\{g_{i} \in G \mid e_{i} \neq 0\right\} . G[B]$ is a group considered as a subgroup of the group of units of $B[G]$. Consult the survey article [1] for basic properties of Boolean powers.

A group $G$ is said to be $B$-separating if $G[B] \simeq G\left[B^{\prime}\right]$ implies $B \simeq B^{\prime}$ for all Boolean rings $B$ and $B^{\prime}[1]$.

In this note we prove:

(1) No Abelian group is $B$-separating;

(2) A finite subdirectly irreducible group is $B$-separating if and only if it is non-Abelian. On the other hand, given a group $G$, the group $G \times G$ is not $B$-separating.

It is known that no finite Abelian group is $B$-separating. Neumann and Yamamuro have proved that a countable non-Abelian simple group is $B$-separating [6], while Jonsson has proved that a countable centerless indecomposable group is $B$-separating [3].

After this paper had been submitted, the author discovered that G. Bergman [7] had proved that no Abelian group is $B$-separating. Because of its simplicity, we have retained our original proof of this fact.

If $B$ is a Boolean ring, then there is a partial order $<$ on $B$ defined by $e<f$ if $e f=e$. Now consider $B$ as a vector space over $Z_{2}$, the two-element field. We say that $B$ has a totally ordered basis if $B$ has a totally ordered basis (under $<$ ) as a vector space over $Z_{2}$. It is well known that every countable Boolean ring has a totally ordered basis [5].

Received by the editors February 20, 1980.

1980 Mathematics Subject Classification. Primary 06E99, 20E10; Secondary 08B99.

Key words and phrases. Boolean power, Boolean ring, group.

${ }^{1}$ Research was supported by a Grant from NSERC. 
LEMMA 1. Let $B$ be a Boolean ring with a totally ordered basis $T=\left\{t_{i}\right\}_{i \in e}$ and let $G$ be an Abelian group. Then an element $x$ of $G[B]$ has a unique representation of the form

$$
x=\left[\left(1-t_{1}\right)+t_{1} g_{1}\right]\left[\left(1-t_{2}\right)+t_{2} g_{2}\right] \cdots\left[\left(1-t_{k}\right)+t_{k} g_{k}\right],
$$

where $t_{1}>t_{2}>\cdots>t_{k}, g_{i} \neq 1$, and $k>0$.

Proof. Clearly $\sum_{i=1}^{n} e_{i} g_{i}=\Pi_{i=1}^{n}\left[\left(1-e_{i}\right)+e_{i} g_{i}\right]$, so we need to show that every element of the form $(1-e)+e g$ has a representation of the form (*). Suppose $e=t_{1}+t_{2}+\cdots+t_{k}$, where $t_{1}>t_{2}>\cdots>t_{k}$. Then

$$
\begin{aligned}
& {[(1-e)+e g]=\left[\left(1-t_{1}\right)+t_{1} g\right]\left[\left(1-t_{2}\right)+t_{2} g^{-1}\right]\left[\left(1-t_{3}\right)+t_{3} g\right] } \\
& \cdots\left[\left(1-t_{k}\right)+t_{k} g^{(-1)^{k+1}}\right] .
\end{aligned}
$$

We have proved that every element of $G[B]$ has a representation of the form (*).

To show that this representation is unique, we must show that if $k>0$, then $x \neq 1$. But $t_{1}\left(1-t_{2}\right) \cdots\left(1-t_{k}\right)=t_{1}\left(1-t_{2}\right) \neq 0$, so if $k>0, g_{1}$ occurs in the support, hence $x \neq 1$.

TheOREM 2 (BERgMAN [7]). No Abelian group is B-separating.

Proof. Let $B$ and $B^{\prime}$ be two nonisomorphic Boolean rings with the same cardinality and totally ordered bases, $\left\{t_{i}\right\}_{i \in \mathcal{C}}$ and $\left\{s_{i}\right\}_{i \in \mathcal{C}}$ respectively. Let $G$ be an Abelian group. Let $\alpha$ be a bijection from the set $\left\{t_{i}\right\}_{i \in \mathcal{C}}$ to the set $\left\{s_{i}\right\}_{i \in \mathcal{C}}$. Define

$$
\phi\left[\left(1-t_{i}\right)+t_{i} g\right]=\left[\left(1-\alpha\left(t_{i}\right)\right)+\alpha\left(t_{i}\right) g\right]
$$

and extend this multiplicatively. It is easily proved, using Lemma 1 , that $\phi$ extends to an isomorphism from $G[B]$ to $G\left[B^{\prime}\right]$. Since $B \cong B^{\prime}$, while $G[B] \simeq G\left[B^{\prime}\right]$, we have shown that $G$ is not $B$-separating.

Theorem 2 can be generalized to show that no affine algebra [4] is $B$-separating.

Let $G$ be a non-Abelian finite subdirectly irreducible $p$-group with $|G|=p^{n}$. Let $C$ be the center of $G, K$ be the minimal nontrivial normal subgroup of $G$ and $A$ be the second element in the upper central series of $G$. Let $B$ be a Boolean ring. For each $x \in A[B]$, let

$$
G_{x}=\left\{x^{(1)} x^{(2)} \cdots x^{\left(p^{n} s\right)} \mid x^{(i)} \text { a conjugate } x \text { in } G[B], s>1\right\} .
$$

Let $K_{x}=K[B] \cap G_{x}$.

Lemma 3. Suppose $x=\sum x_{g} g \in A[B]$. Then we have

$$
K_{x}=\left\{\sum_{g \in K} y_{g} g \mid \sum_{\substack{g \neq 1 \\ g \in K}} y_{g}<\sum_{\substack{g \in A \\ g \notin C}} x_{g}\right\} .
$$

Hence, $K_{x} \supset K_{x^{\prime}}$ if and only if

$$
\Sigma\left\{x_{g} \mid g \in A, g \notin C\right\}>\Sigma\left\{x_{g}^{\prime} \mid g \in A, g \notin C\right\} .
$$


Proof. We first prove that $K_{x} \supset\left\{\Sigma_{g \in K} y_{g} g \mid \Sigma_{g \neq 1, g \in K} y_{g}<\Sigma_{g \in A, g \notin C} x_{g}\right\}$. Let $e=e_{g h}=y_{h} x_{g}, h \in K, g \in A \backslash C$. Let $t \in G$ be an element such that $t^{-1} g t=a g$, $1 \neq a \in C$. For some power of $a$, say $m, a^{m}=h$ (by the subdirect irreducibility of $G)$. Then $t^{-m} g t^{m}=a^{m} g=h g$. Now,

$$
x^{p^{n-1}}[(1-e)+e t]^{-m} x[(1-e)+e t]^{m}=(1-e)+e h \in K_{x} .
$$

Hence,

$$
\prod_{\substack{g \in A \backslash C \\ h \in K}}\left[\left(1-e_{g h}\right)+e_{g h} h\right]=\sum_{h \in K} y_{h} h,
$$

and this completes the first part of the proof

We now show that $K_{x} \subset\left\{\sum_{g \in K} y_{g} g \mid \sum_{g \neq 1, g \in K} y_{g}<\Sigma_{g \in A, g \notin C} x_{g}\right\}$. Let $y=$ $\Sigma_{g \in K} y_{g} g$ be an element of $K_{x}$. Then $y$ is a product of $p^{n} s$ conjugates of $x$ (for some $s)$. If $g$ is an element of $C$, then $g^{p_{s}}=1$, thus $x_{g}<y_{1}$. Since $K \subset A$, we have that $\sum\left\{y_{g} \mid g \in K-\{1\}\right\} \leqslant \Sigma\left\{x_{g} \mid g \in A, g \notin C\right\}$.

This completes the proof of the lemma.

Now let $g$ be a subdirectly irreducible finite group with nontrivial center $C$ and suppose that $G$ is not a $p$-group. Since $G$ is subdirectly irreducible, the minimal nontrivial normal subgroup $K$ is a cyclic $p$-group for some prime $p$, and its center has order $p^{m}$ for some $m>1$. Let $q$ be a prime different from $p$, dividing the order of $G$. Let $H$ be the set of elements of $G[B]$ of order some power of $q$. For each $x \in H$, let $\langle x\rangle$ be the principal normal subgroup of $G[B]$ generated by $x$. Finally, define

$$
K_{x}=K[B] \cap\langle x\rangle .
$$

Lemma 4. Suppose $x=\Sigma_{g \in G} x_{g} g \in H$. Then

$$
K_{x}=\left\{\sum_{g \in K} y_{g} g \mid \sum_{\substack{g \in K \\ g \neq 1}} y_{g}<\sum_{\substack{g \in G \\ g \neq 1}} x_{g}\right\} .
$$

Hence, $K_{x} \supset K_{x^{\prime}}$ if and only if

$$
\Sigma\left\{x_{g} \mid g \in G-\{1\}\right\}>\Sigma\left\{x_{g}^{\prime} \mid g \in G-\{1\}\right\} .
$$

Proof. We first prove that

$$
K_{x} \supset\left\{\sum_{g \in K} y_{g} g \mid \sum_{\substack{g \in K \\ g \neq 1}} y_{g}<\sum_{\substack{g \in G \\ g \neq 1}} x_{g}\right\} .
$$

Let $e=e_{g h}=y_{h} x_{g}, h \in K, 1 \neq g \in G$. Since $K \subset\langle g\rangle \subset G$ (by subdirect irreducibility), there is a product of conjugates of $g$ equal to $h$, say

$$
t_{1}^{-1} g t_{1} \cdots t_{l}^{-1} g t_{l}=h, \quad t_{i} \in G .
$$

Then $\Pi_{i=1}^{l}\left[(1-e)+e t_{i}^{-1}\right] x\left[(1-e)+e t_{i}\right]=e h+\sum x_{g}^{\prime \prime} g$, where each $g$ occurring in the sum has order some power of $q$. Since the order of $h$ is a power of $p$, we have

$$
(1-e)+e h \in\langle x\rangle \text {. }
$$


The product

$$
\Pi\left\{\left(1-e_{g h}\right)+e_{g h} h \mid h \in K, g \in \operatorname{supp} x, g \neq 1\right\}=\Sigma\left\{y_{n} h \mid h \in K\right\} .
$$

This completes the first part of the proof.

We now show that $K_{x} \subset\left\{\Sigma_{g \in K} y_{g} g \mid \sum_{g \in K, g \neq 1} y_{g}<\Sigma_{g \in G, g \neq 1} x_{g}\right\}$. The element $x$ has ordered some power of $q$. Let $y=\sum y_{g} g \in K_{x}$. Then $y$ is a product of conjugates of $x$. Thus $y_{1}>x_{1}$. It follows that $\Sigma_{g \in K, g \neq 1} y_{g}<\Sigma_{g \in G, g \neq 1} x_{g}$, since $\Sigma_{g \in G} x_{g}=1$.

This completes the proof of the lemma.

THEOREM 5. Let $G$ be a finite subdirectly irreducible non-Abelian group. Then $G$ is $B$-separating.

Proof. If $G$ is centerless, then the result follows from [3].

If $G$ is a $p$-group, use Lemma 3. Since $A[B]$ is the second element in the upper central series, it can be identified in $G[B]$. By Lemma 3, the partially ordered set $\left\{K_{x} \mid x \in A[B]\right\}$ is isomorphic to $B$. Thus we can 'recover' $B$ from $G[B]$, and this shows that $G$ is $B$-separating.

If $G$ is not a $p$-group, we use Lemma 4 . Then the partially ordered set $\left\{K_{x} \mid x \in H\right\}$ is isomorphic to $B$, hence $G$ is $B$-separating.

REMARK. It seems probable that the subdirect irreducibility in Theorem 5 can be replaced by indecomposability.

LemMa 6. Let $B$ be $a$ Boolean ring and let $G$ and $H$ be groups. Then $(G \times H)[B]$ $\simeq G[B] \times H[B]$.

Proof. Define $\phi:(G \times H)[B] \rightarrow G[B] \times H[B]$ by

$$
\phi\left(\sum_{i} e_{i}\left(g_{i} \times h_{i}\right)\right)=\sum_{i} e_{i} g_{i} \times \sum_{i} e_{i} h_{i}
$$

This is an isomorphism with inverse $\phi^{-1}$ defined by

$$
\phi^{-1}\left(\sum_{i} e_{i} g_{i} \times \sum_{j} f_{j} h_{j}\right)=\sum_{i, j} e_{i} f_{j}\left(g_{i} \times h_{j}\right) .
$$

Proposition 7. Let $G$ be a group. Then the group $G \times G$ is not B-separating.

Proof. Let $B$ and $B^{\prime}$ be nonisomorphic Boolean rings such that $B \times B \cong B^{\prime} \times$ $B^{\prime}$ [2]. Then

$$
\begin{aligned}
(G \times G)[B] & \cong G[B] \times G[B] \cong G[B \times B] \cong G\left[B^{\prime} \times B^{\prime}\right] \\
& \cong G\left[B^{\prime}\right] \times G\left[B^{\prime}\right] \cong(G \times G)\left[B^{\prime}\right] .
\end{aligned}
$$

Since $B \approx B^{\prime}$, we have shown that $G \times G$ is not $B$-separating.

\section{REFERENCES}

1. S. Burris, Boolean powers, Algebra Universalis 5 (1975), 341-360.

2. W. Hanf, On some fundamental problems concerning isomorphism of Boolean algebras, Math. Scand. 5 (1957), 205-217. 
3. B. Jonsson, On isomorphic types of groups and other algebraic systems, Math. Scand. 5 (1957), 224-229.

4. R. McKenzie, Para primal varieties: a study of finite axiomatizability and definable principal congruences in locally finite varieties, Algebra Universalis 8 (1978), 336-348.

5. A. Mostrowski and A. Tarski, Boolesche Ringe mit geordneter Basis, Fund. Math. 32 (1939), 69-86.

6. B. H. Neumann and S. Yamamuro, Boolean powers of simple groups, J. Austral. Math. Soc. 5 (1965), 315-324.

7. G. Bergman, Boolean rings of projection maps, J. London Math. Soc. (2) 4 (1972), 593-598.

Department of Pure Mathematics, University of Waterloo, Waterloo, Ontario, Canada N2L 3G1 